\title{
Survey of Panaromic Radiographic Examination of Edentulous Jaws Prior To Denture Construction
}

\author{
P.Sesha Reddy MDS ${ }^{\mathrm{a}}$, R. Pradeep $\mathrm{MDS}^{\mathrm{b}}$, Ashish.R.Jain MDS ${ }^{\mathrm{c}}$, C.J .Venkata \\ Krishnan MDS ${ }^{\mathrm{d}}$ \\ ${ }^{a}$ Reader, ${ }^{b}$ Reader, ${ }^{c}$ Senior Lecturer,${ }^{d}$ Professor and head, ,Department of Prosthodontics, Tagore Dental College \\ And Hospitals, Chennai, India.
}

\begin{abstract}
SOURCE: Department of Prosthodontics, Crown \& Bridge, and Implantology, Tagore Dental College and Hospital, Rathinamangalam, Vandalur Post,Chennai-600127, India.www.tagoredch.com

BACKGROUND: Panoramic radiography is a valuable diagnostic tool in the pretreatment assessment of partially and completely edentulous patients.Many investigators recommended routine radiographic examination for the edentulous jaws before denture treatment, but their routine use in such patients is still not confirmed.

AIM: The present study was undertaken to review the significance of pre-treatment panoramic radiographic assessment of edentulous patients, in the dental patient population of Chennai, India.

MATERIALS AND METHODS: A survey of 705 panoramic radiographs in a span of 36 months was selected from the patient at Tagore Dental College and Hospitals, Chennai,India.Radiographs were evaluated for the presence of seven entities; root fragments, impacted teeth, radiolucencies, radiopacities, foreign bodies, Mental foramina near the crest of the residual ridge, Maxillary sinus close to the crest of the residual ridge. The chi-square test of goodness of fit for equal proportion was utilized to determine the difference of radiographic entities.

RESULTS: Of the 705 patients included in the study,48.36\% Patients $(n=341)$ were already wearing denture and $51.63 \%(n=364)$ were newly edentulous patients with a mean age of 51.5 years. Of 705 patients $70.92 \%$ patients $(n=500)$ were free of positive findings and $29.07 \%$ patients $(n=205)$ have shown positive findings. Out of 205 patients $51.21 \%(n=105)$ were already wearing dentures and $48.78(n=100)$ were newly edentulous patients, $56.09 \%(n=115)$ were males and $43.09 \%(n=90)$ were females. Out of 205 cases, 45 patients $(21.95 \%)$ had one or more remaining root fragments. 26 patients (12.68\%) had impacted teeth. The radiographs of 9 patients (4.39\%) demonstrated radiopacities, and 13 patients $(6.34 \%)$ demonstrated radiolucencies. The radiographs of 6 patients (2.92\%) exhibited foreign bodies.42 patients $(20.48 \%)$ mental foramina was near the crest of the residual alveolar ridge.64 patients(31.21\%) maxillary sinus was close the crest of the residual alveolar ridge. Application of the Chi-square test has shown that root fragments, maxillary sinus and mental foramen was a highly significant entity in comparison to the other entities.
\end{abstract}

CONCLUSION: The results of this and previous studies demonstrate the necessity of routine radiographic examination of the jaws for all edentulous patients before constructing complete dentures. It also indicates the need for a better extraction technique together with the use of radiographs before and after extraction.

Key words: Panoramic radiography, edentulous patients, mental foramen, embedded teeth, retained root stumps,Maxillary sinus.

\section{Introduction:}

Maximum benefits from dentures can only be achieved through denture's stability and retention. It can only be achieved if there is good foundation support, so the supporting bone must be free from all of the intrabony and soft tissue pathosis. The early diagnosis of such abnormalities located in the jaws, which are otherwise asymptomatic, can only be achieved through radiographic examination because alveolar ridges of an edentulous patient covered by a healthy looking mucosa are usually not suspected of harboring any abnormality. The need for radiographic examination of edentulous patients seeking replacement dentures was first reported by Logan and Eusterman in $1921^{1,2}$.Panoramic Radiography is of special value in the diagnosis and treatment planning for the removable Prosthodontic patient. It provides a view of entire maxillomandibular region on a single film without inconveniencing the patient and with minimal time expenditure. It also helps to detect the pathologic changes in otherwise healthy jaws that were missed out during routine clinical examination. A variety of radiographic techniques were recommended including intra-oral, extra-oral and panoramic surveys 3,4 . However, no technique per se can completely satisfy all the requirements for an accurate shadow casting ${ }^{5,6}$ In spite of its obvious diagnostic advantage, such a routine examination is currently being questioned because of 
the cumulative effects of radiation exposure and the limited influence of panoramic examination on surgical and Prosthodontic treatment plans.

AIM: The present study was undertaken to review the significance of pre-treatment panoramic radiographic assessment of edentulous patients, in the dental patient population of Chennai, India.

\section{Material And Methods}

The protocol of this study was revised and approved by the Department of Prosthodontics, Crown \& Bridge, and Implantology, Tagore Dental College and Hospital, Chennai, India. Written informed consent was obtained from those who agreed to participate for performing radiographic examination. All patients are routinely screened with panoramic radiographs before complete denture treatment. A frame of all panoramic radiographs (Orthopantomograph10E, Siemens) taken from 2008 to 2011 was made, the total number of radiographs were 1538 .

Inclusion Criteria for patients were

$>$ Between 39 years to 72 years of age.

$>$ Edentulous in one or both arches

$>$ Edentulous for at least 2 years

$>$ Previous denture experience or correction in one or both arches and were seeking fabrication of a new denture

Exclusion Criteria

$>$ Poor quality radiographs

$>$ Patients under radiation therapy

Considering both the inclusion and exclusion criteria for this study, 833 radiographs were omitted. Therefore the final sample size was 705 radiographs of both sexes ( 373 males and 332 female patients).None of the patients have been reported to possess pathological signs or symptoms or clinical evidence of pathosis as revealed by their clinical examination and reported in their files.

Panoramic radiograph was taken using Panoramic machine (Rota graph plus, panoramic and cephalometric machine, VILLA SISTEMI MEDICALI, 2002, Made in Italy) with $\mathrm{kVp}$ of $60-85$ and standard $10 \mathrm{~mA}$ provided with a total filtration of $2.5 \mathrm{~mm}$ aluminium. Exposure parameters selected were $65 \mathrm{kVp}$ and $10 \mathrm{~mA}$. Exposure time was standard 17 seconds for adults. X ray films used were 5X12 inches Kodak TMAT green light sensitive panoramic dental films made in U.S.A. by Eastman Kodak, Rochester, New York in a 5X12 inches curved rigid aluminium cassette with green light sensitive intensifying screens (Konika KR-II).Then all the radiographs were meticulously examined under standard conditions of radiographic interpretation of a dim room lighting, blockage of film periphery by black papers, using a viewer with even distribution of light and with variable light intensity and using a magnifying glass and were evaluated by the authors and an oral radiology specilaist using dental viewing box without any magnification. The radiographs were evaluated for the presence of

$>$ Retained root fragments,

$>$ Impacted teeth,

$>$ Radiolucencies,

$>$ Radiopacities

$>$ Foreign bodies

Location of the mental foramina at or near the crest of the residual alveolar ridge.

$>$ Maxillary sinus proximity to the crest of the residual alveolar ridge.

The vertical ramus, mental foramen, maxillary tuberosity, zygomatic process and canine fossa were used as anatomical landmarks for recognizing the tooth to which the retained root belonged.

\section{STATISTICAL ANALYSIS}

All data were collected and analysed using descriptive studies. The chi-square test of goodness of fit for equal proportion was utilized to determine the difference of radiographic entities.

Level of significance @ 5\% (0.05)

Power@90\%

\section{Results}

Of the 705 patients included in the study, $48.36 \%$ patients $(\mathrm{n}=341)$ were already wearing denture and $51.63 \%(\mathrm{n}=364)$ were newly edentulous patients with a mean age of 51.5 years of which $52.90 \%(\mathrm{n}=373)$ were men [53\% ( $\mathrm{n}=198)$ were wearing dentures and $46.91 \%(\mathrm{n}=175)$ were newly edentulous patient] and $47.09 \%$ 
$(n=332)$ were women [43.07\% $(n=143)$ were already wearing denture and $56.92 \%(n=189)$ were newly edentulous patient]. Accordingly this study included 1326 edentulous arches $(n=705)$ of which $88.08 \%(n=621)$ were completely edentulous, $7.51 \%(n=53)$ were edentulous in maxiila and $4.39 \%(n=31)$ were edentulous in mandible. Of 705 patients $70.92 \%(n=500)$ patients were free of positive findings $90.2 \%(n=451)$ were completely edentulous, $6.6 \%(n=33)$ were edentulous in maxilla only and $3.2 \%(n=16)$ were edentulous in mandible only and $29.07 \%(n=205)$ patients have shown positive findings of which $82.92 \%(n=170)$ were completely edentulous, 9.75\% ( $\mathrm{n}=20)$ were edentulous in maxilla and $7.31 \%(\mathrm{n}=15)$ were edentulous in mandible. Out of 205 patients $51.21 \%(n=105)$ were already wearing dentures, $48.78 \%(n=100)$ were newly edentulous patients $56.09 \%(n=115)$ were males and $43.09 \%(n=90)$ were females. Out of $29.07 \%(n=205)$, $21.95 \%(n=45)$ patients had one or more remaining root fragments, $12.68 \%(n=26)$ had impacted teeth, $4.39 \%$ $(\mathrm{n}=9)$ demonstrated radiopacities, 6.34\% $(\mathrm{n}=13)$ demonstrated radiolucencies, $2.92 \%(\mathrm{n}=6)$ exhibited foreign bodies, $20.48 \%(n=42)$ mental foramina was near the crest of the residual alveolar ridge, $31.21 \%(n=64)$ maxillary sinus was close the crest of the residual alveolar ridge. Application of the Chi-square test has shown that root fragments, maxillary sinus and mental foramen was a highly significant entity in comparison to the other entities. (Table 1)

\section{Root stumps}

A total of 78 root stumps were found in $(n=45)$ patients. Single root was found in 27 patients, two roots were found in 9 patients and 3 roots were found in 5 patients. Maximum number of root stumps found was four which were found in 4 patients. Out of 45 positive cases $37.77 \%(n=17)$ were females and $62.22 \%(n=28)$ were males. Out of 45 cases having retained root stumps $68.88 \%(n=31)$ were already wearing dentures and $31.11 \%$ $(n=14)$ were newly edentulous patients. Most of the roots were located in maxillary left quadrant $(n=12)$ followed by maxillary right quadrant $(n=12)$, mandibular left quadrant $(n=11)$ and mandibular right quadrant $(n=10)$. Statistically there was no significant difference found in the ratio of retained root stumps in all the four quadrants.

\section{Embedded teeth}

A total of 34 embedded teeth were found in $(n=26)$ patients. Single embedded tooth was found in 17 patients and two embedded teeth were found in 7 patients. Maximum number of embedded teeth found was three which were found only in 2 patients. Out of 26 positive cases $38 \%(n=10)$ were females and $62 \%(n=16)$ were males. Out of 26 positive cases $50 \%(n=13)$ were already wearing dentures and $50 \%(n=13)$ were newly edentulous patients. Most of the embedded teeth were located in the mandibular left quadrant $(n=11)$ followed by mandibular right quadrant $(n=8)$ and maxillary right quadrant $(n=8)$. Maxillary left quadrant was found to harbor 8 embedded teeth. Out of 19 embedded teeth in mandible 15 were present in molar region which was significant statistically with $\mathrm{p}$ value of less than 0.001 .4 embedded teeth were found in canine region. Out of 15 embedded teeth in maxilla 13 were present in molar region which was significant statistically with $\mathrm{p}$ value of less than 0.0003. 2 embedded teeth were found in canine region.

\section{Radiolucencies}

A total of 13 radiolucencies were found in 13 patients. Out of 13 positive cases $46.2 \%$ ( $n=6)$ were females and $53.8 \%(n=7)$ were males. Out of 13 positive cases there were $38.5 \%(n=5)$ already wearing dentures and $61.5 \%(n=8)$ were newly edentulous patients. Out of these 13 radiolucencies 3 were diagnosed as residual cysts, 2 as Stafne bone cyst, 1 as carcinoma of alveolus, 2 as residual infection and 5 as osteoporotic areas.

\section{Radiopacities}

A total of 9 radiopacities were found in 9 patients. Out of 9 positive cases $66.66 \%(n=6)$ were females and $33.33 \%(n=3)$ were males. Out of 9 positive cases $44.44 \%(n=4)$ were already wearing dentures and $55.55 \%$ $(\mathrm{n}=5)$ were newly edentulous patients. Out of these 9 radiopacities 3 were diagnosed as osteosclerosis, 2 as calcifications of soft tissues like lymph nodes, tonsils, lips and alveolar mucosa, 2 as polyp in maxillary sinus, 2 as mucous retention cyst in maxillary sinus.

\section{Foreign bodies}

A total of 6 foreign bodies were found in 6 patients. Out of 6 positive cases $33.33 \%(n=2)$ were females and $66.66 \%(n=4)$ were males. Out of 6 positive cases $66.66 \%(n=4)$ were already wearing dentures and $33.33 \%$ $(n=2)$ were newly edentulous patients.Out of these 6 positive cases 3 were diagnosed as amalgam particle, 2 as endodontic files, and 1case was not diagnosed. 


\section{Mental foramen at the crest of residual alveolar ridge}

In patients the mental foramen was situated at the crest of the residual alveolar ridge. Out of 42 positive cases $42.85 \%(n=18)$ were females and $57.14 \%(n=24)$ were males. Out of 42 positive cases $54.76 \%(n=23)$ were already wearing dentures and $45.23 \%(n=19)$ were newly edentulous patients. Statistically the difference in the ratio of mental foramen at the crest of the residual alveolar ridge among denture wearers and newly edentulous patients was significant with a p-value of 0.019 .

\section{Maxillary sinus was close the crest of the residual alveolar ridge.}

In patients the maxillary sinus was situated at the crest of the residual alveolar ridge. Out of 64 positive cases $45.31 \%(\mathrm{n}=29)$ were females and $54.68 \%(\mathrm{n}=35)$ were males. Out of 64 positive cases $39.06 \%(\mathrm{n}=25)$ were already wearing dentures and $60.93 \%(n=39)$ were newly edentulous patients. Statistically the difference in the ratio of maxiilary sinus at the crest of the residual alveolar ridge among denture wearers and newly edentulous patients was significant with a p-value of 0.024 .

Distribution of positive findings among male and females and among denture wearers and non denture wearers is shown in (Fig 1 and Fig.2) respectively.

\section{Discussion}

Most edentulous persons have lost their teeth due to various dental or periodontal conditions. Despite that the teeth are extracted and the ridges have healed in a satisfactory manner, hidden lesions or infections may not have been totally eradicated. ${ }^{7}$ This study demonstrated and emphasized the need for a radiographic examination of the edentulous patients before constructing complete dentures for them. This is of great benefit because the normal appearance of the dental ridges may conceal problem underneath. This view is in acceptance with the views presented by other investigators ${ }^{8,9}$.

The panoramic radiographic examination of 705 completely edentulous patients revealed positive radiographic findings in $29.07 \%$. This study shows that there is almost equal prevalence of positive radiographic findings in completely edentulous patients in Chennai, India, like other parts of the world. Comparison of the results of the present study with the previous ones is tabulated in (Table 2). Our study has shown that majority of the patients with positive radiographic findings $51.21 \%(\mathrm{n}=105)$ were already wearing dentures and $48.78 \%$ $(\mathrm{n}=100)$ were newly edentulous patients while Lyman $\mathrm{S}$ et al in 1990 discouraged the routine radiographic examination of patients who were already wearing complete denture as he found only one embedded tooth that required extraction out of total 150 already denture wearing patients ${ }^{10}$ Out of total 78 root stumps in $(n=45)$ patients. Most of the roots were located in maxillary left quadrant $(n=12)$ followed by maxillary right quadrant $(\mathrm{n}=12)$, mandibular left quadrant $(\mathrm{n}=11)$ and mandibular right quadrant $(\mathrm{n}=10)$. Most of the retained roots are found in the molar region of the maxilla.The reason may be attributed to the wrong technique of extraction and not using the radiographs before and after the extraction regularly. Another important aspect is the quadrant in which retained root stumps are present. In our study majority of the retained root stumps are found in maxillary right and left quadrant $(n=12)$.Here it is important to note that many reports did not record the side of disclosure. We emphasize on the need of careful extraction in maxillary left and right quadrant. Removal of retained root stumps is a controversial subject ${ }^{11}$ Until there is associated pathologic change root stumps can be retained to preserve the integrity of the edentulous ridges but as they serve as a potential source of infection should be extracted as soon as possible. Otherwise the patient should be informed of all such situations so that the dentist is not held responsible for any complication that may arise in future. But follow up examinations and radiographs should be done at regular intervals and findings should be entered into the patient's record. The relationship of mental foramen and maxillary sinus to the crest of the residual alveolar ridge is very important. If mental foramen exits directly at the crest of the residual alveolar ridge it can lead to pain or numbness in the area due to compression caused by denture. In present study $20.48 \%(\mathrm{n}=42)$ of patients the mental foramen was situated at the crest of the residual alveolar ridge. Out of 42 positive cases $54.76 \%(n=23)$ were already wearing dentures and $45.23 \%(\mathrm{n}=19)$ were newly edentulous patients and $32.21 \%(\mathrm{n}=64)$ the maxillary sinus was situated at the crest of the residual alveolar ridge, out of 64 positive cases $39.06 \%(\mathrm{n}=25)$ were already wearing dentures and $60.93 \%(n=39)$ were newly edentulous patients Statistically the difference in the ratio of mental foramen and maxillary sinus at the crest of the residual alveolar ridge among denture wearers and newly edentulous patients was statistically significant. The reason can be attributed to the fact that the occlusal forces are transmitted directly to the mucosa beneath the mucosa-borne dentures and can cause overloading, which might lead to bone resorption and shift in the position of the maxillary sinus and mental foramen to the tip of the residual ridge.

The present study reported the prevalence of positive radiographic findings in panoramic radiographs of asymptomatic patients. Three of the categories of findings were clearly identified, but two were given only the general descriptive terms of radiolucencies and radiopacities without diagnostic interpretation. Because most of the radiolucencies and radiopacities have no recorded impact on treatment planning but it is in the interest of 
both the patient and the dentist to be aware of the presence of such innocuous findings even if the treatment is required or not. Furthermore, due to difficulties in differential radiographic interpretation, radiolucencies and radiopacities should be further evaluated clinically and radiographically. Biopsies should be made if a lesion appears suspicious, or it should be observed periodically to detect possible changes in location or size that could endanger the patient's health or affect the fit of the denture. Although we did not assess the impact of their observations on treatment, we can say that the high incidence of positive findings reemphasize the need for radiographic examination of all edentulous. Our results refute the view of those dentists who downplay the importance of panoramic radiographs and make prostheses for patients after a brief clinical examination. The reasons may be attributed to the high cost of panoramic radiographs and/or clinically healthy looking edentulous arches but our study has shown high incidence of positive findings in healthy looking edentulous arches. Another concern may be the radiation dose but due to utilization of the rare earth screens, fast films and the digital radiography radiation dose to patient from panoramic radiographic examination is extremely low, so can be used with minimal risk ${ }^{12}$. Finally we suggest that for a successful prosthodontic treatment pre-treatment panoramic radiographs should be made mandatory in order to ensure the delivery of the highest quality of care to each of its individual patients.

\section{Conclusion}

The results of this and previous studies demonstrate the necessity of routine radiographic examination of the jaws for all edentulous patients before constructing complete dentures. It also indicates the need for a better extraction technique together with the use of radiographs before and after extraction.

\section{References}

[1]. Logan, W.H. G.: Should all pulpless teeth be removed? J. Natl. Dent. Assoc., 8: 126 131, 1921

[2]. Eusterman, M. F.: Roentgenographic findings in two hundred and ninety partially edentulous or edentulous mouths. Dent cosmos. 63: 902 903, 1921

[3]. Swenson, H. M.: Roentgenographic examination of the edentulous mouth J. Am. Dent. Assoc., 31: $475478,1944$.

[4]. Swenson, H. M., and Hudson, J. R.: Roentgenographic examination of edentulous patients. J. Prosthet. Dent. $18: 403$-407, 1967.

[5]. John, D.J., Richard, R., and Ernst, S.: Panoramic Radiographic examination of edentulous patients. J. Prosthet. Dent. 53: 535 - 539, 1985.

[6]. Axelsson, C.O.: Orthopantomographic examination of the edentulous mouth. J. Prosthet.Dent. 59: 592 - 598, 1988.

[7]. Richard R.S, Earl O.W., and John D.J.: Panoramic Radiographs Necessary for Patients, JADA.123:74 -78, 1992.

[8]. Sanford L. and Luis J. B.: Radiographic examination of edentulous mouths J. Prosthet. Dent. 64: 180 - 182, 1990.

[9]. Susan, M. W. The radiographic examination of edentulous patients, J. Prosthet Dent. 50: 164-166, 1983.

[10]. Lyman S, Boucher LJ. Radiographic examination of edentulous mouths. J Prosthet Dent.1990;64:180-2.

[11]. Manson-Hing, L.R. Fundamentals of dental radiography, Ed, Philadelphia,1979, Lea \& Febiger, P.169, 1985.

[12]. Keur JJ. Radiographic screening of edentulous patients: Sense or Nonsense? A risk - benefit analysis. Oral Surg Oral Med Oral Pathol. 1986;62:463-7.

TABLE I: Number And Percent Of Radiographic Entities

\begin{tabular}{|c|c|c|}
\hline RADIOGRAPHIC ENTITY & NO OF CASES & $\begin{array}{c}\text { \% OF RADIOGRAPHIC } \\
\text { ENTITIES }\end{array}$ \\
\hline Root fragments & 45 & $21.95 \%$ \\
\hline Impacted teeth/embedded teeth & 26 & $12.68 \%$ \\
\hline $\begin{array}{c}\text { Radiolucencies } \\
\text { And mixed lesions }\end{array}$ & 13 & $6.34 \%$ \\
\hline Radiopacities & 09 & $2.39 \%$ \\
\hline Foreign bodies & 06 & $20.48 \%$ \\
\hline $\begin{array}{c}\text { Mental foramina near the crest of } \\
\text { the residual ridge }\end{array}$ & 42 & $31.21 \%$ \\
\hline $\begin{array}{c}\text { Maxillary sinus close to the crest } \\
\text { of the residual ridge. }\end{array}$ & 64 & \\
\hline Total & 205 & \\
\hline
\end{tabular}


TABLE II: COMPARISON OF RESULTS OF PRESENT STUDY WITH THE PREVIOUS STUDIES

\begin{tabular}{|l|c|c|c|c|}
\hline $\begin{array}{c}\text { NAME OF THE } \\
\text { AUTHOR }\end{array}$ & $\begin{array}{c}\text { REPORTING } \\
\text { YEAR }\end{array}$ & $\begin{array}{c}\text { RADIOGRAPHIC } \\
\text { TECHNIQUE } \\
\text { USED }\end{array}$ & $\begin{array}{c}\text { NUMBER OF } \\
\text { PATIENTS } \\
\text { EXAMINED }\end{array}$ & $\begin{array}{c}\text { \% OF } \\
\text { POSITIVE } \\
\text { CASES }\end{array}$ \\
\hline Logan & 1921 & NOT REPORTED & 35 & 28.6 \\
\hline Eusterman & 1921 & NOT REPOETED & 290 & 38.3 \\
\hline Cook & 1927 & NOT REPORTED & 500 & 38.0 \\
\hline Gardner \& stafne & 1929 & NOT REPORTED & 2112 & - \\
\hline Swenson \& hudson & 1967 & PAN & 400 & 18.0 \\
\hline Perrelet et al & 1977 & PAN & 287 & 41.1 \\
\hline Serman \& Nortje & 1982 & PAN & 539 & 47.1 \\
\hline Jones et al & 1985 & PAN & 114 & 34.4 \\
\hline Angulo F & 1989 & PAN & 200 & 38.5 \\
\hline Edgerton et al & 1991 & PAN & 308 & 23.0 \\
\hline Seals RR et al & 1992 & PAN & 448 & 11.6 \\
\hline Mehdizade et al & 2005 & PAN & 192 & - \\
\hline Masood et al & 2007 & PAN & 327 & 42.5 \\
\hline Sumer et al & 2007 & PAN & 338 & 47.6 \\
\hline Ardakani et al & 2007 & PAN & 447 & - \\
\hline Present study & $\mathbf{2 0 0 8 - 2 0 1 1}$ & PAN & $\mathbf{7 0 5}$ & $\mathbf{2 9 . 0 7}$ \\
\hline
\end{tabular}

PAN-Panaromic radiographs

FIGURE 1: DISTRIBUTION OF POSITIVE FINDINGS AMONG MALES AND FEMALES.

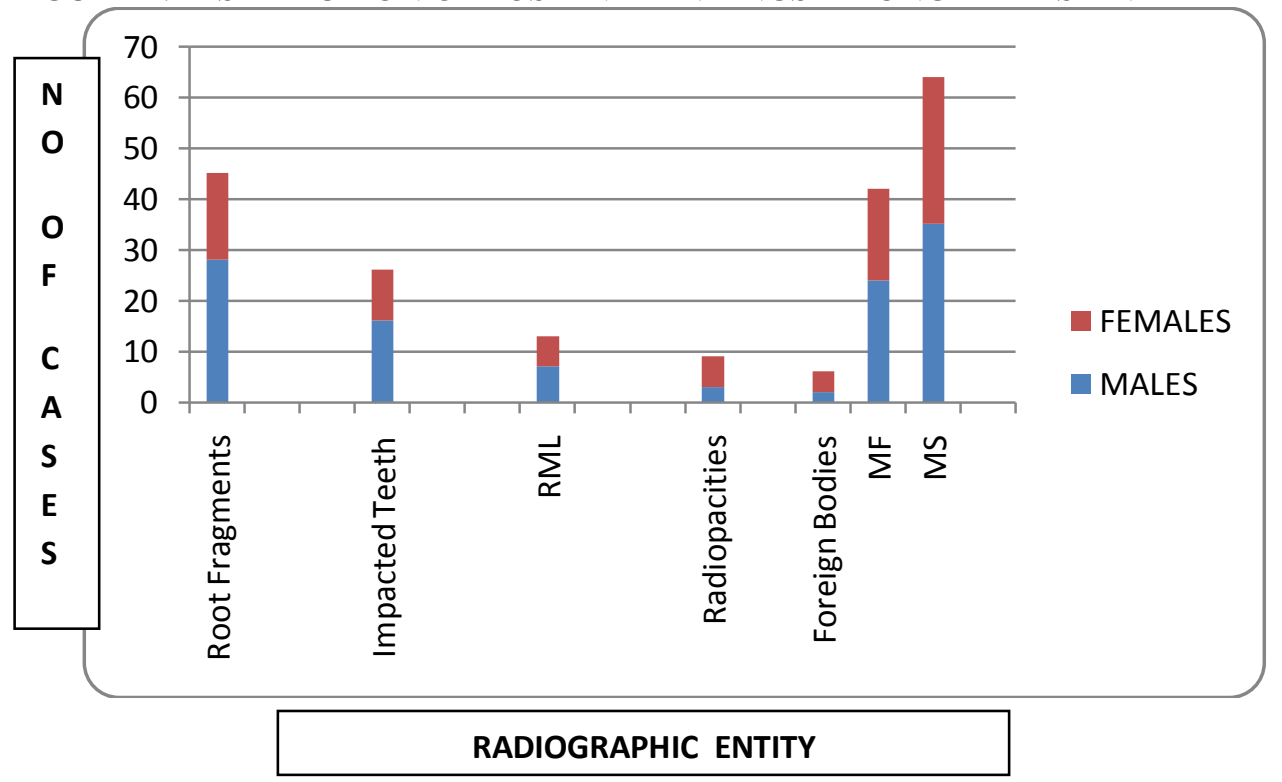

RML- Radioluciencies And Mixed Dentition

MF- Mental foramen Close To Residual Alveolar Ridge

MS- Maxillary Sinus Close To Residual Alveolar Ridge 
FIGURE 2: DISTRIBUTION OF POSITIVE FINDINGS AMONG DENTURE WEARERS AND NEWLY EDENT ULOUS PATIENTS.

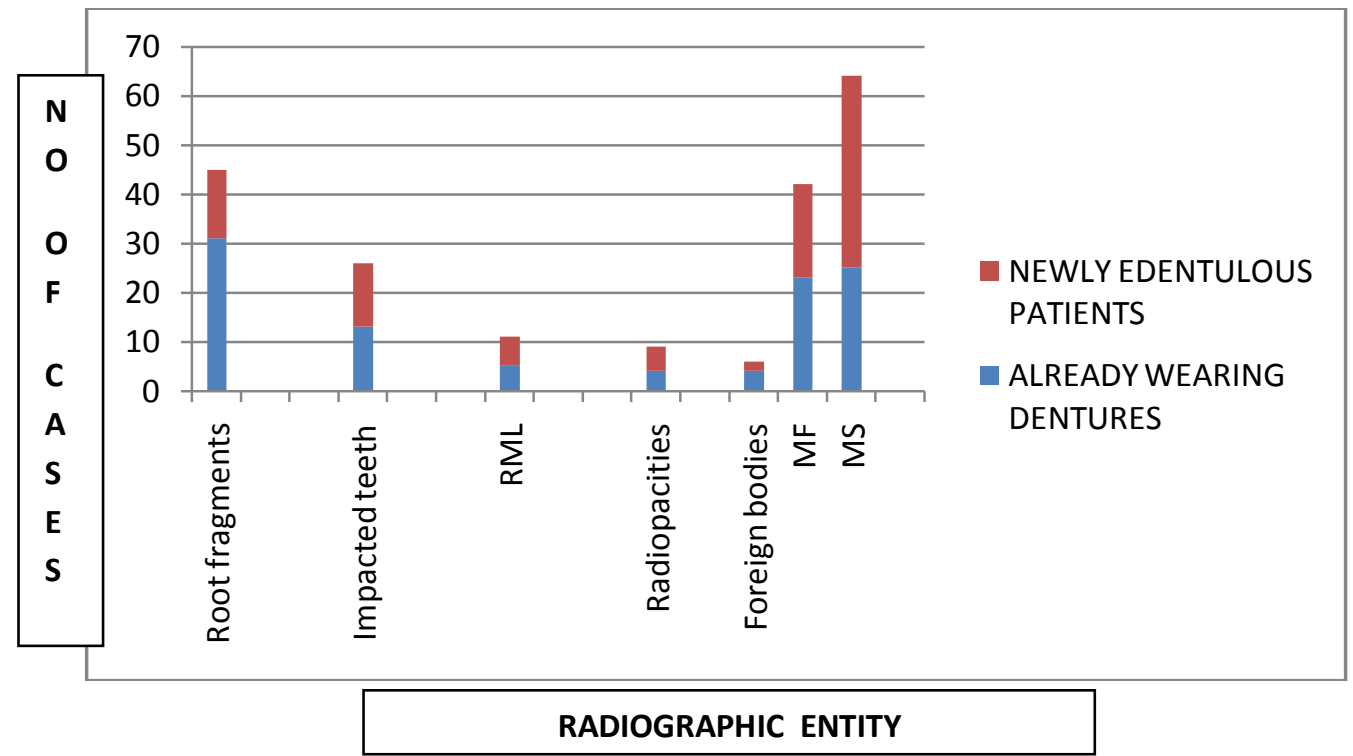

RML- Radioluciencies And Mixed Dentition

MF- Mental foramen Close To Residual Alveolar Ridge

MS- Maxillary Sinus Close To Residual Alveolar Ridge 